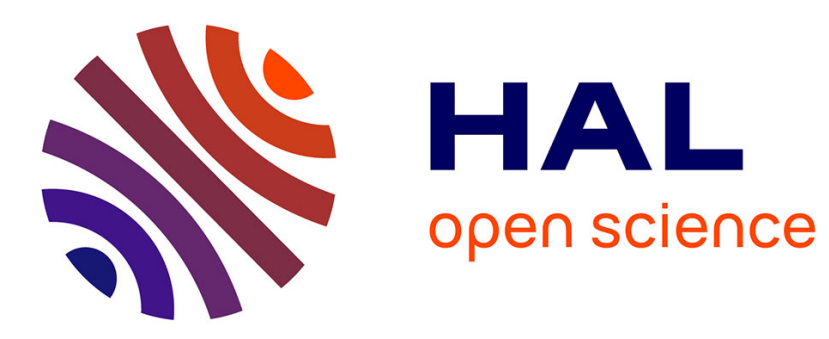

\title{
Experimental investigation of short pulse Raman amplification with backward pumping
}

Guillaume Vanderhaegen, Pascal Szriftgiser, Matteo Conforti, Alexandre Kudlinski, Maxime Droques, Arnaud Mussot

\section{To cite this version:}

Guillaume Vanderhaegen, Pascal Szriftgiser, Matteo Conforti, Alexandre Kudlinski, Maxime Droques, et al.. Experimental investigation of short pulse Raman amplification with backward pumping. Optics Letters, 2021, 46 (19), pp.5019-5022. 10.1364/OL.434956 . hal-03374878

\author{
HAL Id: hal-03374878 \\ https://hal.science/hal-03374878
}

Submitted on 12 Oct 2021

HAL is a multi-disciplinary open access archive for the deposit and dissemination of scientific research documents, whether they are published or not. The documents may come from teaching and research institutions in France or abroad, or from public or private research centers.
L'archive ouverte pluridisciplinaire HAL, est destinée au dépôt et à la diffusion de documents scientifiques de niveau recherche, publiés ou non, émanant des établissements d'enseignement et de recherche français ou étrangers, des laboratoires publics ou privés. 


\title{
Experimental investigation of short pulse Raman amplification with backward pumping
}

\author{
Guillaume Vanderhaegen ${ }^{1, *}$, Pascal Szriftgiser $^{1}$, Matteo Conforti $^{1}$, Alexandre \\ Kudlinski $^{1}$, Maxime Droques ${ }^{2}$, And Arnaud Mussot ${ }^{1}$ \\ ${ }^{1}$ University of Lille, CNRS, UMR 8523 - PhLAM-Physique des Lasers Atomes et Molécules, F-59000 Lille, France \\ ${ }^{2}$ Alcatel Submarine Networks, 950, Quai de la Loire, 62225 Calais Cedex, France \\ *Corresponding author: guillaume.vanderhaegen@univ-lille.fr \\ Compiled May 19, 2021
}

We report an experimental study about the backwardpumped Raman amplification of short pulses into a 20.3 $\mathrm{km}$ long optical fiber. We demonstrate that the gain and the pump saturation depend on the pulse duration. We also reveal that for short enough pulses the amplification process remains linear and very high peak powers, even larger than the Raman pump, are achievable. Numerical simulations reproduces the experimental results with an excellent agreement. () 2021 Optical Society of America

\section{http://dx.doi.org/10.1364/ao.XX.XXXXXX}

With the development of high-bit-rate fiber optic networks in long spans, it was necessary to develop optical amplifiers to compensate the losses suffered by optical data during their transmission through optical fibers. Two types of amplifiers entered into competition: lumped amplifiers such as Erbium doped fiber amplifiers (EDFA) and distributed ones such as Raman amplifiers. After the demonstration of Raman amplification in silica fibers [1, 2], the development of amplifiers based on this process were first investigated in the 80 s, offering promising results with the capability of long haul transmissions [3]. Despite the poor pump power efficiency of Raman amplifiers compared to EDFA which was then preferred, the arrival of high-power pump lasers in the 90s made Raman amplifiers attractive again. They have been used in many systems, including undersea communication channels [4], amplification of picosecond pulses [5], extreme events generation [6], or even loss compensation for fundamental investigation [7-9]. Indeed, Raman amplifiers offer the advantage of a wide bandwidth gain [10], which can even be broaden by cascading additional shifted pumps [11]. Moreover, its distributed feature allows to actively compensate the intrinsic losses during the propagation, providing a better signal to noise ratio than lumped amplifiers [12]. To minimize the relative intensity noise transfer from the pump, they are preferably used in the backward pumped configuration [13]. In the continuous wave regime, the dynamics of both forward and backward configurations can be described analytically [14]. However in the pulsed regime, analytical solutions are found in the forward pumped configuration only. The backward interaction was solved in the case of negligible losses only [15, 16]. Remarkably in [16] gigan- tic amplification of short pulses and pump saturation effects are noticed. To the best of our knowledge, no experimental study has been performed yet highlighting short pulse amplification and amplifier saturation induced distortion. In this letter, we report this experimental investigation of the saturated regime of short pulse amplification in a backward-pumped Raman amplifier.

The amplification of a pulsed Stokes wave induced by a counter-propagating Raman pump is governed by the two following coupled propagation equations [12]:

$$
\begin{gathered}
\frac{\partial A_{S}}{\partial z}+\frac{1}{v_{g, S}} \frac{\partial A_{S}}{\partial t}=\frac{g_{R}}{2}\left|A_{P}\right|^{2} A_{S}-\frac{\alpha_{S}}{2} A_{S} \\
-\frac{\partial A_{P}}{\partial z}+\frac{1}{v_{g, P}} \frac{\partial A_{P}}{\partial t}=-\frac{\lambda_{S}}{\lambda_{P}} \frac{g_{R}}{2}\left|A_{S}\right|^{2} A_{P}-\frac{\alpha_{P}}{2} A_{P}
\end{gathered}
$$

The indices $S$ and $P$ account respectively for the $1550 \mathrm{~nm}$ Stokes signal wave (which is amplified) and for the backward $1450 \mathrm{~nm}$ Raman pump wave (which is depleted). $A_{S, P}(z, t)$ represent the slowly-varying envelopes of the waves, $v_{g}$ the group velocity, $g_{R}$ the Raman gain coefficient and $\alpha$ the intrinsic loss coefficient. Here, the dispersive effects can be neglected as the dispersion length $L_{D, S / P}$ [17] of the signal and pump waves is much larger than the fiber length. Thus, only the group velocity mismatch has to be taken into account. The amplification scheme implemented being counter-propagative, the boundary conditions of both pump and signal are known at different spatial positions. The signal is known at fiber input-end, $A_{S}(z=0, t)$, while the Raman pump is known at fiber output-end, $A_{P}(z=L, t)$. In this case, for the numerical simulation of Eqs. $(1,2)$, it is convenient to solve by time iterations. An operator splitting is performed, using a Runge-Kutta method of the $4^{\text {th }}$ order to solve the nonlinear step. Moreover, if we assume $v_{g, S}=v_{g, P}=v_{g}$, we can easily solve the linear step by following the characteristics, i.e. considering a temporal step related to the spatial step as $\Delta t=\frac{\Delta z}{v_{g}}$ [18]. This scheme allows to avoid the numerical artifacts caused by sharp fronts encountered in the traditional Fourier split-step method.

A sketch of the typical interaction scenario is presented in Fig. 1 (a). The signal wave (blue curve, travelling at speed $v_{g, S}$, is amplified by the pump wave (red curve), travelling in the opposite direction at the velocity $v_{g, P}$ and which is depleted. An 
example of the space-time power profiles of both Stokes signal pulse and Raman pump obtained from numerical simulations using realistic parameters is shown in Fig. 1 (b,c). A $700 \mathrm{~mW}$ CW Raman pump is launched at the output-end of a $20 \mathrm{~km}$ long optical fiber. To benefit from the maximum gain, the pump is located $13.2 \mathrm{THz}$ away from the spectrum of the signal, a 4 $\mu$ s square pulse of $25 \mathrm{~mW}$. On this example, in Fig. 1 (b) we distinguish the drift of the signal pulse due to the group velocity and its amplification by around $13 \mathrm{~dB}$ between the input and output of the fiber. The Raman pump profile is depicted in Fig. 1 (c). We notice the depletion feature and the attenuation from $z=L$ to $z=0$. It is worth noting that at the fiber output, the signal power is of the same order of magnitude as the initial Raman pump power.
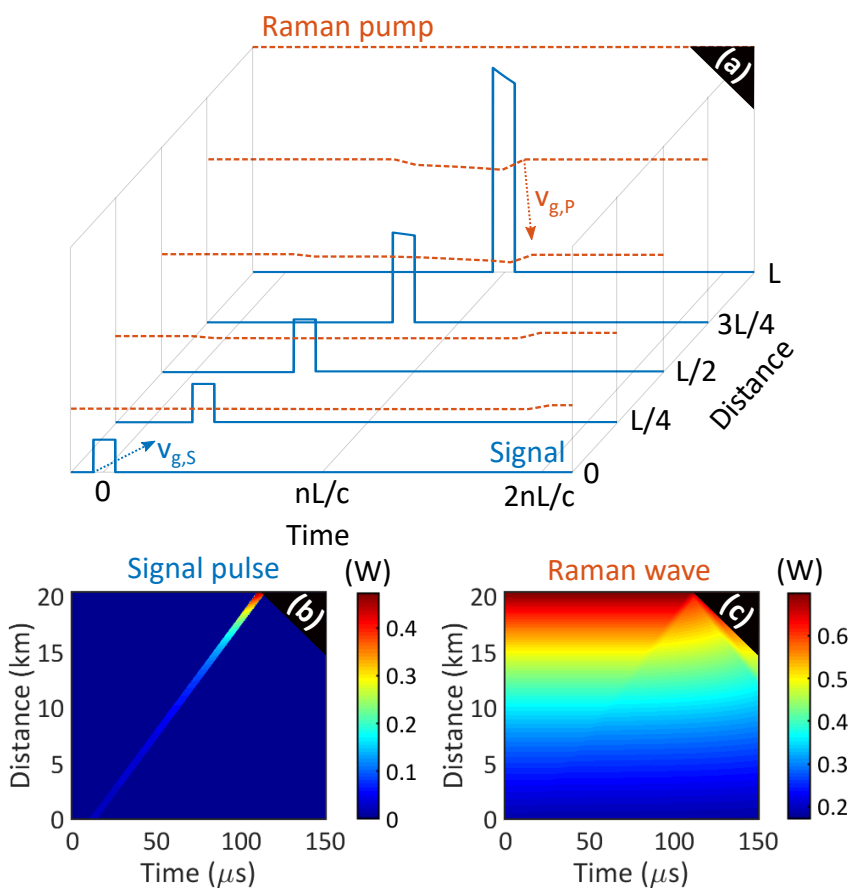

Fig. 1. (a) Space-time scheme of a signal pulse amplified by a backward-pumped Raman amplifier. Numerical spatiotemporal power distributions of both (b) Stokes pulse wave and (c) Raman pump wave. Input parameters: $P_{S}(z=0)=25$ $\mathrm{mW}, T_{S}=4 \mu \mathrm{s}, P_{P}(z=L)=700 \mathrm{~mW}$. Fiber parameters: $\alpha_{S}=0.2 \mathrm{~dB} / \mathrm{km}, \alpha_{P}=0.28 \mathrm{~dB} / \mathrm{km} ; g_{R}=0.49 \mathrm{~W} / \mathrm{km}$.

Backward Raman amplification of Stokes pulses is experimentally investigated using the setup presented in Fig. 2. A $1550 \mathrm{~nm}$ laser generates a CW signal which is first phase modulated to avoid the simulated Brillouin scattering (SBS) [19]. Then, an arbitrary waveform generator (AWG) generates a $4.9 \mathrm{kHz}$ clock which controls RF pulses sequence of a pulse signal generator (PSG). This RF sequence drives an intensity modulator (IM), shaping the $C W$ signal into a $T_{S}$ duration square-shaped pulses train. It is then amplified by an erbium doped fiber amplifier (EDFA) to reach a peak power of $P_{S}(z=0)=25$ $\mathrm{mW}$. The noise level between two consecutive pulses being relatively high $(20-25 \mathrm{~dB}$ extinction ratio from IM and decrease of SNR due to EDFA), the pulses train is time filtered by an AO modulator driven by the same PSG. A typical input pulse is shown in the inset in Fig. 2. It is then injected into a $20.3 \mathrm{~km}$ long non-zero dispersion-shifted fiber (NZDSF) whose specifications are $g_{R}=0.49 \mathrm{~W} / \mathrm{km}, \alpha_{S}=\alpha(@ 1550 \mathrm{~nm})=0.2$

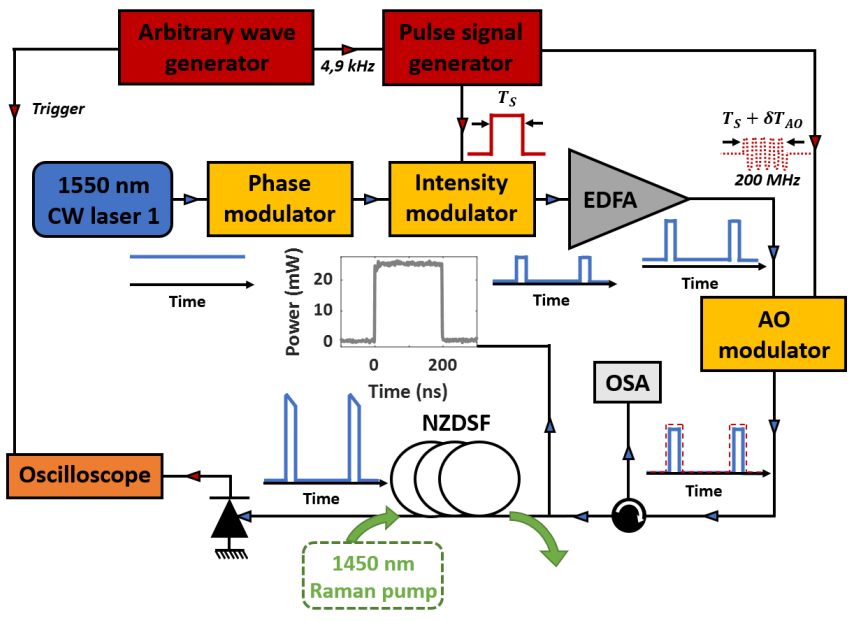

Fig. 2. Sketch of the experimental setup. On the inset is displayed a typical $200 \mathrm{~ns}$ input pulse with $25 \mathrm{~mW}$ peak power.

$\mathrm{dB} / \mathrm{km}, \alpha_{P}=\alpha(@ 1450 \mathrm{~nm})=0.28 \mathrm{~dB} / \mathrm{km}$ and $n=1.47$. The zero-dispersion wavelength is located around $1578.4 \mathrm{~nm}$. The dispersion regime is normal at $1550 \mathrm{~nm}$, preventing to trigger modulation instability which could distort the pulses during their propagation. A counter-propagating Raman pump is implemented to amplify the pulses during their propagation inside the fiber. The Raman pump wave $(1450 \mathrm{~nm})$ is located $13.2 \mathrm{THz}$ away from $1550 \mathrm{~nm}$ signal to benefit from maximum gain. At the fiber output-end, the amplified pulses are captured by a photodiode and displayed on the oscilloscope.

We measured the on-off energy gain at the fiber output i.e. the amplification factor between output pulses when the Raman pump is on and off. The energy is calculated from the recorded pulses temporal traces. First we study the saturation regime of the backward pumped Raman amplifier as a function of the pulses duration. The recordings of amplified Stokes signal pulses are realized for several durations ranging from $10 \mathrm{~ns}$ to $4 \mu \mathrm{s}$. The upper value is limited by the SBS while the lower one by the bandpass of our electronic devices. This range is sufficiently wide to observe the saturation phenomenon. The experiments are realized for three distinct pump power values, $P_{R}=P_{P}(z=L)=703 \mathrm{~mW}, P_{R}=801 \mathrm{~mW}$ and $P_{R}=898 \mathrm{~mW}$, allowing to investigate the dynamic of the process. On the upper panel of Fig. 3 we display the experimental energy gain as a function of the signal pulse duration. In each case the tendency is similar. The gain is almost constant for short pulse durations $(<100 \mathrm{~ns})$ around 50, 86 and 145 for all respective pump power values. As expected from the basic theory in the unsaturated regime, the Raman gain increases with the pump power. Moreover, the measured gain values are in good agreement with the basic theory for $\mathrm{CW}$ operation, which gives $G=\exp \left(g_{R} P_{R} L_{e f f}\right)$ with $L_{e f f}=\frac{1-\exp \left(-\alpha_{P} L\right)}{\alpha_{P}}(49.4,85.0$ and 145.7 respectively) [12]. These features can be explained as follows. When the Stokes signal wave crosses the pump wave, the $1450 \mathrm{~nm}$ pump photons converted into $1550 \mathrm{~nm}$ Stokes photons belong to different pump time slots. For short pulses, this number is negligible compared to the number of pump photons as the time overlap between the pump time slot and the signal pulse is small. As an example in Fig. 3. (b), (e) and (h) we display the power time profiles of the fiber output Stokes pulses with $T_{S}=70 \mathrm{~ns}$ (solid green lines). We notice that regardless of the pump power, the pulses remain 

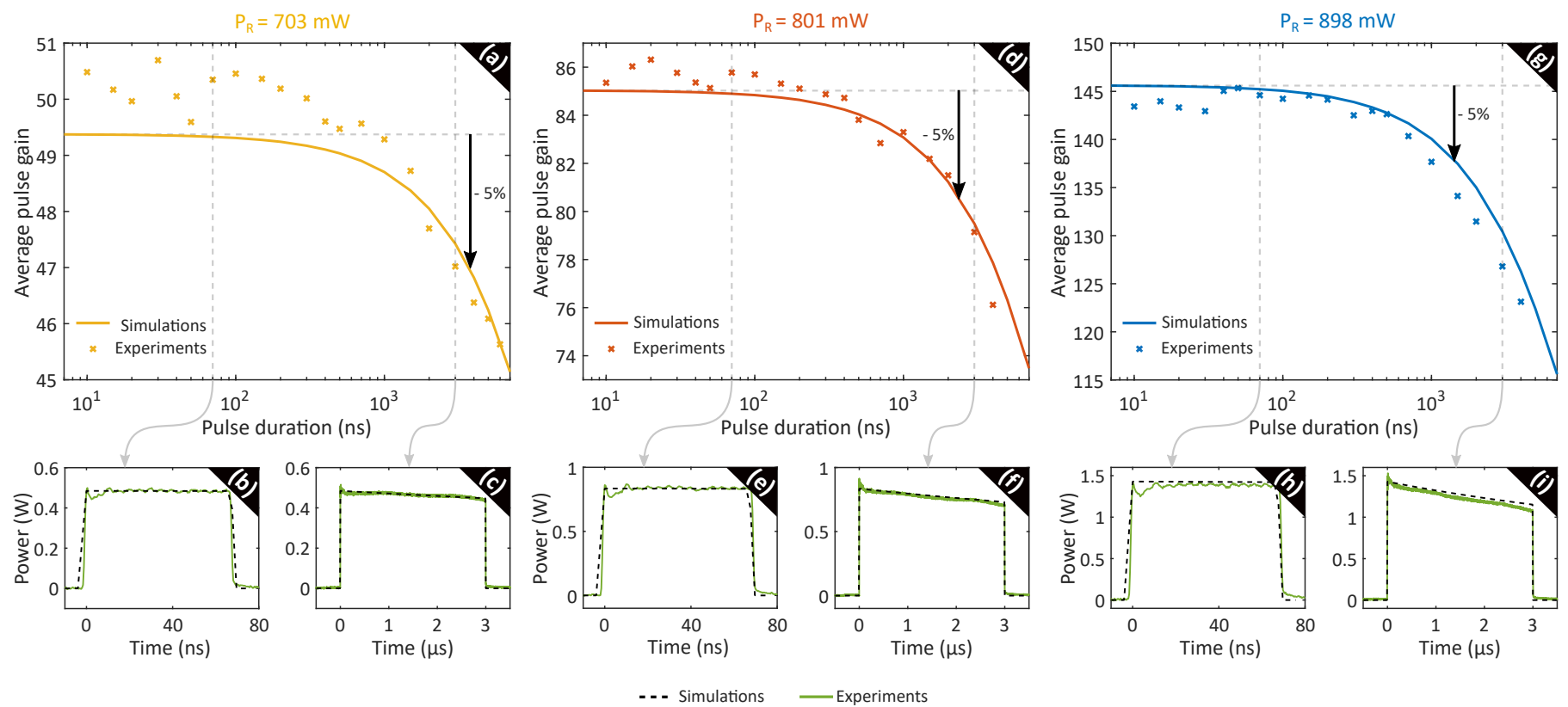

Fig. 3. On-off energy gain as function of the pulse width for a Raman pump power (a) $P_{R}=703 \mathrm{~mW}$, (d) $P_{R}=801 \mathrm{~mW}$ and (g) $P_{R}=898 \mathrm{~mW}$. Experimental data are represented by crosses while numerical data by solid lines. For each Raman pump power, the power time profiles of the amplified Stokes pulses are presented for pulse durations (b),(e),(h) $70 \mathrm{~ns}$ and (c),(f),(i) $3 \mu \mathrm{s}$. Experimental pulse shapes are plotted in solid green line and numerical ones in black dashed line.

in a square shape after amplification, confirming the unsaturated regime. Indeed, the number of depleted pump photons being negligible, the trailing end of the pulse is amplified by the same unsaturated Raman pump than the leading end. The Raman pump never saturates for short pulses, leading to a striking feature: pulses with high output peak powers. For $P_{R}=898 \mathrm{~mW}$, the pulses are even amplified beyond the pump value, reaching power values around $1.4 \mathrm{~W}$. It is more than 1.5 times higher than the pump which would be impossible in the CW regime. For longer pulses, the gain drops due to the saturation process. The crossing time between the pump time slots and the signal pulse is not small anymore, as the pulse durations become larger. The number of converted photons from the pump is thus larger, depleting the pump and leading to a gain drop. As an example in figs. 3. (c), (f) and (i) we display the power time profiles of the fiber output Stokes pulses for $T_{S}=3 \mu \mathrm{s}$ (solid green lines). Distortion due to pump saturation is observed, the pulses trailing ends are less powerful than the leading ends. Indeed, the pulse leading front see an unsaturated Raman pump (output pulses leading front powers are logically equal to $T_{S}=70 \mathrm{~ns}$ output pulses powers) while the trailing edge is amplified by a saturated one. Consequently, the gain is lower at the trailing end (the power decreases by $28 \%$ between leading and trailing edges for $P_{R}=898 \mathrm{~mW}$ ). We also notice that the gain drop appears for shorter pulses when we increase the Raman pump power. Indeed, the $5 \%$ gain drop from the plateau value is reached at $3850 \mathrm{~ns}, 2230 \mathrm{~ns}$ and $1320 \mathrm{~ns}$ for the respective pump powers. Moreover, the power differential between pulse leading and trailing ends on output pulses power time profiles (saturation distortion) is more significant at higher pump power. The saturation effects are more significant at shorter pulse widths when the Raman pump power is increased. These experimental results are compared with numerical simulations of Eqs. $(1,2)$. Numerical data are plotted in solid lines for the gain curves and in black dashed lines for the pulse time profiles. The overall agreement between experiments and numerics is very good on several points. The on-off gain values are very close for each case of Raman pump power and the experimental gain curve trends follow numerical ones. It is worth noting the excellent agreement with the amplified pulses shapes.

For comparison, the forward Raman pump amplification configuration is also investigated. Likewise, the energy gain is displayed for Stokes pulse widths ranging from $10 \mathrm{~ns}$ to $3 \mu \mathrm{s}$ in Fig. 4. (a) (purple crosses). The gain is constant for $T_{S} \geq 50$

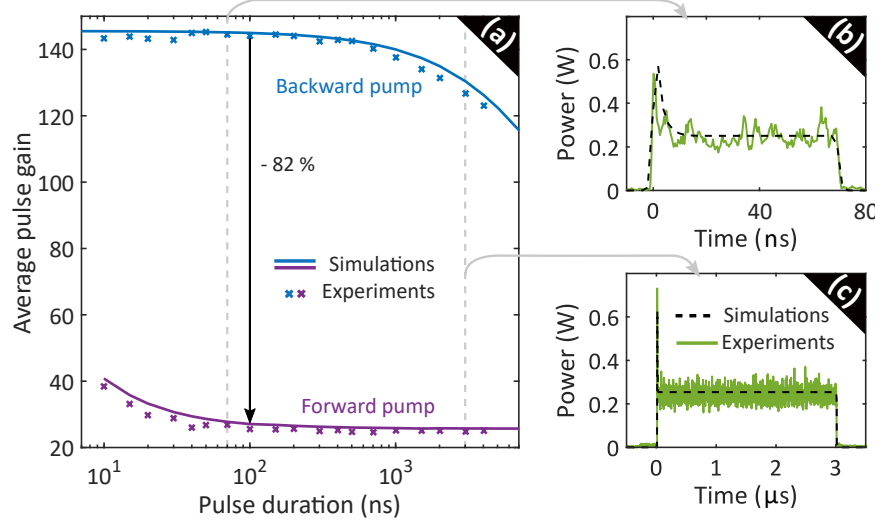

Fig. 4. (a) Energy gain as a function of the pulse duration for a backward (blue) and a frontward (purple) pumping Raman amplification schemes. Experimental data are represented by crosses while numerical data by solid lines. Time profiles of the output co-amplified pulses for (b) $T_{S}=70 \mathrm{~ns}$ and (c) $T_{S}=3 \mu$ s (experiments in green solid lines and numerics in black dashed lines).

ns because the signal and pump waves co-propagate. Indeed, both waves propagate approximately with the same velocity 
(the walk-off is not significant over the pulse duration), so the Stokes pulse is amplified by almost the same pump time slot during all the fiber propagation. The gain is then equal to the saturated gain of the amplifier in the $\mathrm{CW}$ approximation. No pulse width dependency of the gain is thus observed. However, because of the walk-off, in the short pulse regime, a gain increase is noticed. The output forward-amplified pulses time profiles are also displayed in Fig. 4 . (b) and (c) for $T_{S}=70 \mathrm{~ns}$ and $T_{S}=$ $3 \mu$ s respectively. Excepted the noisy fluctuations due to the relative intensity noise (RIN) transfer, the amplified pulses seem to maintain their square shapes. However, both pulses have a few nanoseconds amplification peak at the leading edge, due to the walk-off (the pulse front end shifts away to undepleted time slots of the pump during the propagation). For comparison, on the same figure is also displayed the gain curve for the backward Raman pump configuration (blue crosses). The gain is much weaker with a forward pump by around $82 \%$ at $T_{S}=100 \mathrm{~ns}$. Numerical data are also plotted in a comparison purpose with the experimental results. The velocity mismatch, calculated from the dispersion curve, is $\Delta v_{g}=v_{g, S}-v_{g, P}=39 \mathrm{~km} / \mathrm{s}$.

In conclusion, we have reported the experimental investigation of the saturated regime of a backward-pumped Raman amplifier with pulsed signals. We demonstrated that the gain experienced by pulsed signals depends on the signal duration. For short pulse durations, we observed that the amplifier behaves in the perfectly linear regime. This enable to amplify short signal pulses without amplifier saturation induced distortion and to reach peak powers of the amplified signal possibly larger than the Raman initial power. The experimental results are in excellent agreement with the numerical simulations. In addition to the fundamental aspect, this research brings a better comprehension of Raman amplification of short pulses which may be relevant for some applications. For example, Raman amplification has been used to obtain totally transparent fibers $[7,8]$ which are required for the investigation of some nonlinear effects in optical fibers. It can also be useful in the context of signal amplification in fiber-based sensors and more generally in telecommunication systems.

\section{Funding.}

Disclosures. The authors declare no conflicts of interest.

\section{REFERENCES}

1. R. H. Stolen, E. P. Ippen, and A. R. Tynes, Appl. Phys. Lett. 20, 62-64 (1972).

2. R. G. Smith, Appl. Opt. 11, 2489 (1972).

3. L. F. Mollenauer, R. H. Stolen, and M. N. Islam, Opt. Lett. 10, 229-231 (1985).

4. P. Hansen and L. Eskildsen, Opt. Fiber Technol. 3, 221-237 (1997).

5. C. Finot, G. Millot, C. Billet, and J. M. Dudley, Opt. Express 11, 1547-1552 (2003).

6. K. Hammani, C. Finot, J. M. Dudley, and G. Millot, Opt. Express 16, 16467 (2008).

7. G. Xu, M. Conforti, A. Kudlinski, A. Mussot, and S. Trillo, Phys. Rev. Lett. 118, 254101 (2017).

8. A. Mussot, C. Naveau, M. Conforti, A. Kudlinski, F. Copie, P. Szriftgiser, and S. Trillo, Nat. Photonics 12, 303-308 (2018).

9. G. Vanderhaegen, P. Szriftgiser, A. Kudlinski, M. Conforti, S. Trillo, M. Droques, and A. Mussot, Opt. Express 28, 17773 (2020).

10. J. Bromage, K. Rottwitt, and M. E. Lines, IEEE Photonics Technol. Lett. 14, 24-26 (2002).

11. K. Rottwitt and H. D. Kidorf, "A $92 \mathrm{~nm}$ bandwidth raman amplifier," in Optical Fiber Communication Conference, (Optical Society of America, 1998), p. PD6.
12. C. Headley and G. Agrawal, Raman Amplification in Fiber Optical Communication Systems, Electronics \& Electrical (Elsevier Science, 2005).

13. C. R. S. Fludger, V. Handerek, and R. J. Mears, J. Light. Technol. 19, 1140 (2001).

14. M. Santagiustina, Opt. Lett. 32, 3023-3025 (2007).

15. M. Maier, W. Kaiser, and J. A. Giordmaine, Phys. Rev. 177, 580-599 (1969).

16. V. V. Kozlov, S. V. Manakov, and S. Wabnitz, Opt. Lett. 36, 1632 (2011).

17. G. Agrawal, Nonlinear Fiber Optics (Academic Press, 2007).

18. C. Sun, N. Mangan, M. Dong, H. G. Winful, S. T. Cundiff, and J. Nathan Kutz, J. Opt. Soc. Am. B 36, 3263 (2019).

19. Y. Aoki, K. Tajima, and I. Mito, J. Light. Technol. 6, 710-719 (1988). 


\section{FULL REFERENCES}

1. R. H. Stolen, E. P. Ippen, and A. R. Tynes, "Raman oscillation in glass optical waveguide," Appl. Phys. Lett. 20, 62-64 (1972).

2. R. G. Smith, "Optical power handling capacity of low loss optical fibers as determined by stimulated raman and brillouin scattering," Appl. Opt. 11, 2489 (1972).

3. L. F. Mollenauer, R. H. Stolen, and M. N. Islam, "Experimental demonstration of soliton propagation in long fibers: loss compensated by raman gain," Opt. Lett. 10, 229-231 (1985).

4. P. Hansen and L. Eskildsen, "Remote amplification in repeaterless transmission systems," Opt. Fiber Technol. 3, 221-237 (1997).

5. C. Finot, G. Millot, C. Billet, and J. M. Dudley, "Experimental generation of parabolic pulses via raman amplification in optical fiber," Opt. Express 11, 1547-1552 (2003).

6. K. Hammani, C. Finot, J. M. Dudley, and G. Millot, "Optical rogue-wavelike extreme value fluctuations in fiber raman amplifiers," Opt. Express 16, 16467-16474 (2008).

7. G. Xu, M. Conforti, A. Kudlinski, A. Mussot, and S. Trillo, "Dispersive dam-break flow of a photon fluid," Phys. Rev. Lett. 118, 254101 (2017).

8. A. Mussot, C. Naveau, M. Conforti, A. Kudlinski, F. Copie, P. Szriftgiser, and S. Trillo, "Fibre multi-wave mixing combs reveal the broken symmetry of fermi-pasta-ulam recurrence," Nat. Photonics 12, 303-308 (2018).

9. G. Vanderhaegen, P. Szriftgiser, A. Kudlinski, M. Conforti, S. Trillo, M. Droques, and A. Mussot, "Observation of four fermi-pasta-ulamtsingou recurrences in an ultra-low-loss optical fiber," Opt. Express 28, 17773-17781 (2020).

10. J. Bromage, K. Rottwitt, and M. E. Lines, "A method to predict the raman gain spectra of germanosilicate fibers with arbitrary index profiles," IEEE Photonics Technol. Lett. 14, 24-26 (2002).

11. K. Rottwitt and H. D. Kidorf, "A $92 \mathrm{~nm}$ bandwidth raman amplifier," in Optical Fiber Communication Conference, (Optical Society of America, 1998), p. PD6.

12. C. Headley and G. Agrawal, Raman Amplification in Fiber Optical Communication Systems, Electronics \& Electrical (Elsevier Science, 2005).

13. C. R. S. Fludger, V. Handerek, and R. J. Mears, "Pump to signal rin transfer in raman fiber amplifiers," J. Light. Technol. 19, 1140 (2001).

14. M. Santagiustina, "Exact integral solution of saturation and depletion in forward and backward optical fiber raman amplifiers," Opt. Lett. 32, 3023-3025 (2007).

15. M. Maier, W. Kaiser, and J. A. Giordmaine, "Backward stimulated raman scattering," Phys. Rev. 177, 580-599 (1969).

16. V. V. Kozlov, S. V. Manakov, and S. Wabnitz, "Exact solution for the gigantic amplification of ultrashort pulses in counterpumped raman amplifiers," Opt. Lett. 36, 1632 (2011).

17. G. Agrawal, Nonlinear Fiber Optics (Academic Press, 2007).

18. C. Sun, N. Mangan, M. Dong, H. G. Winful, S. T. Cundiff, and J. Nathan Kutz, "Stable numerical schemes for nonlinear dispersive equations with counter-propagation and gain dynamics," J. Opt. Soc. Am. B 36, 3263 (2019).

19. Y. Aoki, K. Tajima, and I. Mito, "Input power limits of single-mode optical fibers due to stimulated brillouin scattering in optical communication systems," J. Light. Technol. 6, 710-719 (1988). 